\title{
Test of the Kugo-Ojima Confinement Criterion in the Lattice Landau Gauge
}

\author{
Hideo Nakajima ${ }^{\mathrm{a}}$ and Sadataka Furui ${ }^{\mathrm{b}}$ \\ ${ }^{a}$ Department of Information Science, Utsunomiya University, \\ 2753 Ishii, Utsunomiya 321-8585 Japan (e-mail nakajima@is.utsunomiya-u.ac.jp) \\ ${ }^{\mathrm{b}}$ School of Science and Engineering, Teikyo University, \\ 1-1 Toyosatodai, Utsunomiya 320-8551, Japan (e-mail furui@dream.ics.teikyo-u.ac.jp)
}

\begin{abstract}
We present the first results of numerical test of the Kugo-Ojima confinement criterion in the lattice Landau gauge. The Kugo-Ojima criterion of colour confinement in the BRS formulation of the continuum gauge theory is given by $u_{b}^{a}(0)=-\delta_{b}^{a}$, where

$\int d x e^{i p(x-y)}\left\langle 0\left|T D_{\mu} c^{a}(x) g\left(A_{\nu} \times \bar{c}\right)^{b}(y)\right| 0\right\rangle=\left(g_{\mu \nu}-\frac{p_{\mu} p_{\nu}}{p^{2}}\right) u_{b}^{a}\left(p^{2}\right)$.

We measured the lattice version of $u_{b}^{a}(0)$ in use of $1 /(-\partial D(A))$ where $D_{\mu}(A)$ is a lattice covariant derivative in the new definition of the gauge field as $U=e^{A}$. We obtained that $u_{b}^{a}(0)$ is consistent with $-c \delta_{b}^{a}, c=0.7$ in $S U(3)$ quenched simulation data of $\beta=5.5$, on $8^{4}$ and $12^{4}$. We report the $\beta$ dependence and finite-size effect of $c$.
\end{abstract}

\section{INTRODUCTION}

The colour confinement problem in the continuum gauge theory was extensively analysed in use of the BRS formulation by Kugo and Ojima国.

The QCD lagrangian is invariant under the BRS transformation and the physical space is specified as the one that satisfies the condition $\mathcal{V}_{\text {phys }}=\{\mid$ phys $\left.\rangle\right\}$

$Q_{B} \mid$ phys $\rangle=0$.

where

$Q_{B}=\int d^{3} x\left[B^{a} D_{0} c^{a}-\partial_{0} B^{a} \cdot c^{a}+\frac{i}{2} g \partial_{0} \bar{c}^{a} \cdot(c \times c)^{a}\right]$ and $(F \times G)^{a}=f_{a b c} F^{b} G^{c}$.

Under the assumption that BRS singlets have positive metric, it is proved that $\mathcal{V}_{\text {phys }}$ has positive semidefinite in such a way that BRS quartet particles appear only in zero norm.

One finds from the BRS transformation that for each colour $a$, a set of massless asymptotic fields $\chi^{a}, \beta^{a}, \gamma^{a}, \bar{\gamma}$ form a BRS quartet.

The Noether current corresponding to the conservation of the colour symmetry is $g J_{\mu}^{a}=$ $\partial^{\nu} F_{\mu \nu}^{a}+\left\{Q_{B}, D_{\mu} \bar{c}\right\}$, where its ambiguity by divergence of antisymmetric tensor should be understood, and this ambiguity is utilised so that massless contribution may be eliminated for the charge, $Q^{a}$, to be well defined.

Denoting $g\left(A_{\mu} \times \bar{c}\right)^{a} \rightarrow u_{b}^{a} \partial_{\mu} \bar{\gamma}^{b}$, and then $D_{\mu} \bar{c}^{a} \rightarrow(1+u)_{b}^{a} \partial_{\mu} \bar{\gamma}^{b}$, one obtains the eq. $\left.{ }^{*}\right)$ provided $A_{\mu}$ has a vanishing expectation value. The current $\left\{Q_{B}, D_{\mu} \bar{c}\right\}$ contains the massless component, $(1+u)_{b}^{a} \partial_{\mu} \beta^{b}(x)$. We can modify the Noether current for colour charge $Q^{a}$ such that

$g J_{\mu}^{\prime a}=g J_{\mu}-\partial^{\nu} F_{\mu \nu}^{a}=\left\{Q_{B}, D_{\mu} \bar{c}\right\}$.

In the case of $\mathbf{1}+\mathbf{u}=\mathbf{0}$, massless component in $g J_{0}^{\prime}$ is vanishing and the colour charge

$Q^{a}=\int d^{3} x\left\{Q_{B}, g^{-1} D_{0} \bar{c}^{a}(x)\right\}$

\section{becomes well defined.}

The physical state condition $Q_{B}|p h y s\rangle=0$ together with the equation (1i) implies that all BRS singlet one particle states $|f\rangle \in \mathcal{V}_{\text {phys }}$ are colour singlet states. This statement implies that all coloured particles in $\mathcal{V}_{\text {phys }}$ belong to BRS quartet 
and have zero norm. This is the colour confinement.

\section{LATTICE CALCULATION OF $u_{b}^{a}$}

The Faddeev-Popov operator is

$\mathcal{M}[U]=-(\partial \cdot D(A))=-(D(A) \cdot \partial)$,

where the new definition of the gauge field is adopted as $U=e^{A}$, and the lattice covariant derivative $D_{\mu}(A)=\partial_{\mu}+A d\left(A_{\mu}\right)$ is given in [2].

The inverse, $\mathcal{M}^{-1}[U]=\left(M_{0}-M_{1}[U]\right)^{-1}$, is calculated perturbatively by using the Green function of the Poisson equation $M_{0}^{-1}=\left(-\partial^{2}\right)^{-1}$ and $M_{1}=\partial_{\mu} A d\left(A_{\mu}(x)\right)$, as

$\mathcal{M}^{-1}=M_{0}^{-1}+\sum_{k=0}^{N_{\text {end }}}\left(M_{0}^{-1} M_{1}\right)^{k} M_{0}^{-1}$.

In use of colour source $\left|\lambda^{a} x\right\rangle$ normalised as $\operatorname{Tr}\left\langle\lambda^{a} x \mid \lambda^{b} x_{0}\right\rangle=\delta^{a b} \delta_{x, x_{0}}$, the ghost propagator is given by

$G^{a b}(x, y)=\left\langle\operatorname{Tr}\left\langle\lambda^{a} x\left|(\mathcal{M}[U])^{-1}\right| \lambda^{b} y\right\rangle\right\rangle$

where the outmost \langle\rangle specifies average over samples $U$.

The ghost propagators of $\beta=5$ and 5.5 are almost the same and they are infrared divergent which can be parameterised as $\frac{1}{p^{2.2}}$. We observed that the ghost propagators of $\beta=6$ is similar to that of $\beta=5.5$ and its finite-size effect is small 3 .

In the similar way, one can calculate the KugoOjima parameter at $p=0$ as,

$$
\begin{aligned}
& \left.\left(g_{\mu \nu}-\frac{p_{\mu} p_{\nu}}{p^{2}}\right) u_{b}^{a}\left(p^{2}\right)\right|_{p=0} \\
= & \left.\left\langle\operatorname{Tr}\left\langle\lambda^{a} p\left|D_{\mu}(A)(\mathcal{M}[U])^{-1}\left(A d\left(A_{\nu}\right)\right)\right| \lambda^{b} p\right\rangle\right\rangle\right|_{p=0}
\end{aligned}
$$

We observed that off-diagonal element of $u_{b}^{a}$ is consistent to zero, but there are statistical fluctuations. The projection operator $g_{\mu \nu}-\frac{p_{\mu} p_{\nu}}{p^{2}}$ in equation $\left(^{*}\right)$ is treated such that it has an expectation value $\frac{3}{4}$ in the limit of $p_{\mu} \rightarrow 0$.

Making the accuracy of the covariant Laplacian equation solver higher, we observe the tendency that the expectation value of $\left|u_{a}^{a}\right|$ increases.

At $\beta=8$, direct measurement of $u_{b}^{a}$ gives a large fluctuation, but suitable $Z_{3}$ twisting treatment for each sample so that the Polyakov scatter plot should be concentrated $\operatorname{around} \arg z=0$,

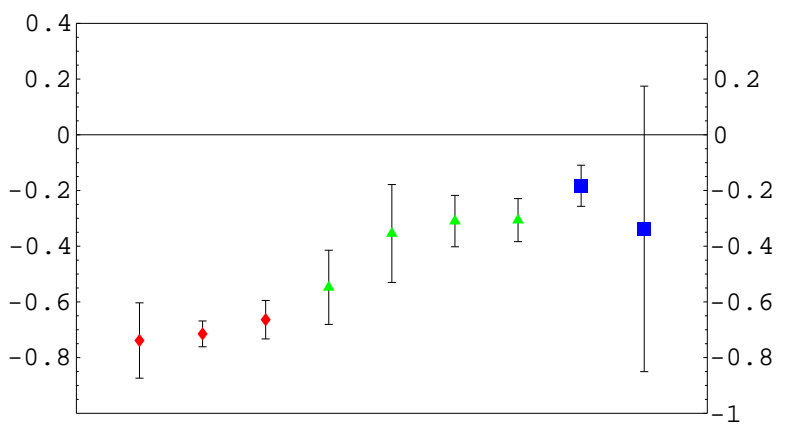

Figure 1. The dependence of space and colour diagonal part of the Kugo-Ojima parameter $u_{a}^{a}$ on $\beta$ and lattice size. (An average over the four directions and eight adjoint representations.) The data points are $\beta=$ $5.5,8^{3} \times 16 ; \beta=5.5,12^{4} ; \beta=5.5,8^{4} ; \beta=$ $6,12^{4} ; \beta=6,8^{4}\left(\right.$ no $\left.Z_{3}\right) ; \beta=6,8^{4}\left(\right.$ with $\left.Z_{3}\right) ; \beta=$ $6,8^{4}$ (with $Z_{3}$, minimum Landau); $\beta=$ $8,8^{4}$ (with $\left.Z_{3}\right) ; \beta=8,8^{4}\left(\right.$ no $\left.Z_{3}\right)$ respectively from left to right.

suppresses the fluctuation and makes the quality of the data better. We consider that this treatment is indispensable in the simulation where $Z_{3}$ symmetry persists and the $Z_{3}$ factor affects the observed quantity. The similar behaviour is observed in $\beta=6,8^{4}$ lattice. The minimum Landau gauge fixing via smeared gauge fixing performed at $\beta=6,8^{4}$ lattice does not change the expectation value obtained after the $Z 3$ twisting but reduces the standard deviation.

The absolute value of $u_{a}^{a}$ is plotted as the function of the spatial extent of the lattice $a L$ where $a$ is calculated by assuming $\Lambda_{\overline{M S}}=100 \mathrm{MeV}$. We find for $a L<2 f m$, there exists large finite-size effectDWe expect that by making $L$ large and $a$ small, such that $a L>2 \mathrm{fm}$, the absolute value of $u_{a}^{a}$ becomes closer to 1 .

Non-symmetric lattice $8^{3} \times 16$ yields nonsymmetric data in $\mu$ of $\left(^{*}\right)$. This fact shows necessity of tuning lattice constants according to the 
Table 1

Kugo-Ojima parameter $u_{a}^{a}$. Space-diagonal $(\mu=\nu)$ and off-diagonal components. All data of $8^{4}$ are the average of 100 samples. 'Z3' and 'min' means Z3 twisting and the minimum Landau gauge fixing.

\begin{tabular}{|c|c|c|c|c|c|c|}
\hline & diag & $f f-\operatorname{diag}$ & $\operatorname{diag}_{1}$ & $\operatorname{diag}_{2}$ & $\operatorname{diag}_{3}$ & $\operatorname{arag}_{4}$ \\
\hline$=$ & $.739(1$ & $0.002(60)$ & .776 & $0.779(105)$ & $0.818(118)$ & $81(49)$ \\
\hline & & & & & & \\
\hline & & & & & & \\
\hline & & & & & & \\
\hline . & & & & & & -( \\
\hline$\beta=6.0$ & -0.308( & -0.0 & 0.3 & -0 & -0 & $2(59)$ \\
\hline$\beta=6.0$, & $-0.354(176)$ & -0.00 & -0.339 & -0.3 & -0.378 & $151)$ \\
\hline$\beta=$ & & 0.0 & & -0.1 & -0.221( & 19) \\
\hline$\beta=8.0,8^{4}$, no $Z_{3}$ & $-0.338(513)$ & $0116(251)$ & $0.264(278)$ & $-0.359(553)$ & $-0.334(610)$ & $-0.394(536)$ \\
\hline
\end{tabular}

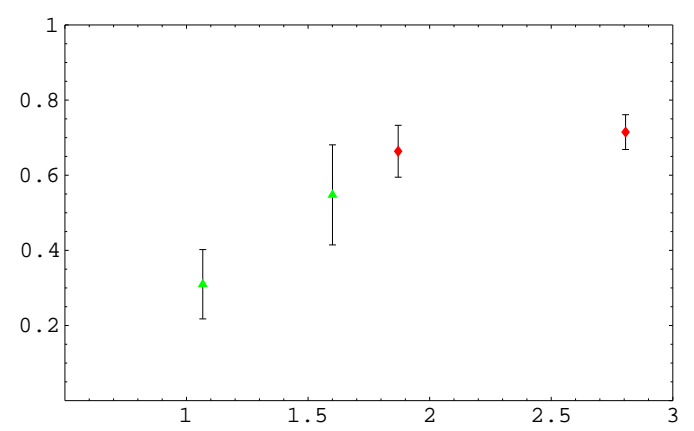

Figure 2. The finite-size effect of the Kugo-Ojima parameter $\left|u_{a}^{a}\right|$ as the function of the spatial extent of the lattice $a L(f m)$.

non-symmetric lattice size and the lattice dynamics.

\section{SUMMARY AND DISCUSSION}

Proof of Kugo-Ojima colour confinement is accomplished successfully only in case of $u_{b}^{a}=-\delta_{b}^{a}$, and this condition is suggested to be a necessary condition as well. We did the first numerical tests of this criterion by the nonperturbative dynamics of lattice Landau gauge. We observed that the value at $\beta=5.5$ is around -0.7 . Its absolute value decreases as $\beta$ increases.

We observed the gluon propagator is infrared finite[2] and the ghost propagator is infrared divergent, suggested to be more singular than $\frac{1}{p^{2}}$, but less singular than $\frac{1}{p^{4}}$. These results qualitatively agree with the Gribov-Zwanziger's conjecture [4, 5], and are consistent with the results of Dyson-Schwinger equation [6]. It is nice to observe that the infrared finiteness of the gluon propagator is in accordance with the Kugo-Ojima colour confinement. As stated in their inverse Higgs mechanism theorem, if we have no massless vector poles in all channels of the gauge field, $A_{\mu}^{a}$, and if the colour symmetry is not broken at all, it follows that $1+u=0$. [7].

This work is supported by High Energy Accelerator Research Organization, KEK Supercomputer Project(Project No.99-46), and by Japan Society for the Promotion of Science, Grant-inaid for Scientific Research(C) (No.11640251).

\section{REFERENCES}

1. T. Kugo and I. Ojima, Prog. Theor. Phys. Supp. 66 (1979) 1

2. H.Nakajima and S. Furui, Nucl. Phys. B,(Proc. Suppl.)73A-C,(1999)635, 865, heplat/9809080,9809081; Confinement III proceedings, hep-lat/9809078

3. H. Suman and K. Schilling, Phys. Lett. B373 (1996) 314.

4. V.N. Gribov, Nucl. Phys. B139 (1978) 1. 
5. D. Zwanziger, Nucl. Phys. B364 (1991) 127, Nucl. Phys. B412 (1994) 657.

6. L. von Smekal, A. Hauck, R. Alkofer, Ann. Phys.267(1998) 1, hep-ph/9707327.

7. K.I. Izawa, hep-th/9411010. 